\title{
Pacific
}

Journal of

Mathematics

\section{MAPS ON INFRA-NILMANIFOLDS}

KyUNG BAI LeE 


\section{MAPS ON INFRA-NILMANIFOLDS}

-Rigidity and applications to Fixed-point Theory

KYUNG BAI LEE

We show that Bieberbach's rigidity theorem for flat manifolds still holds true for any continuous maps on infra-nilmanifolds. Namely, every endomorphism of an almost crystallographic group is semi-conjugate to an affine endomorphism. Applying this result to Fixed-point theory, we obtain a criterion for the Lefschetz number and Nielsen number for a map on infra-nilmanifolds to be equal.

0. Infra-nilmanifolds. Let $G$ be a connected Lie group. Consider the semi-group $\operatorname{Endo}(G)$, the set of all endomorphisms of $G$, under the composition as operation. We form the semi-direct product $G \rtimes \operatorname{Endo}(G)$ and call it aff $(G)$. With the binary operation

$$
(a, A)(b, B)=(a \cdot A b, A B),
$$

the set $\operatorname{aff}(G)$ forms a semi-group with identity $(e, 1)$, where $e \in G$ and $1 \in \operatorname{Endo}(G)$ are the identity elements. The semi-group aff $(G)$ "acts" on $G$ by

$$
(a, A) \cdot x=a \cdot A x .
$$

Note that $(a, A)$ is not a homeomorphism unless $A \in \operatorname{Aut}(G)$. Clearly, aff $(G)$ is a subsemi-group of the semi-group of all continuous maps of $G$ into itself, for $((a, A)(b, B)) x=(a, A)((b, B) x)$ for all $x \in G$. We call elements of $\operatorname{aff}(G)$ affine endomorphisms.

Suppose $G$ is a connected, simply connected, nilpotent Lie group; $\operatorname{Aff}(G)=G \rtimes \operatorname{Aut}(G)$ is called the group of affine automorphisms of $G$. Let $\pi \subset \operatorname{Aff}(G)$ be a discrete subgroup such that $\Gamma=\pi \cap G$ has finite index in $\pi$. Then $\pi \backslash G$ is compact if and only if $\Gamma$ is a lattice of $G$. In this case, $\pi$ is called an almost crystallographic group. If moreover, $\pi$ is torsion-free, $\pi$ is an almost Bieberbach group. Such a group is the fundamental group of an infra-nilmanifold. According to Gromov and Farrell-Hsiang, the class of infra-nilmanifolds coincides with the class of almost flat manifolds. 
1. Generalization of Bieberbach's Theorem. In 1911, Bieberbach proved that any automorphism of a crystallographic group is conjugation by an element of $\operatorname{Aff}\left(\mathbb{R}^{n}\right)=\mathbb{R}^{n} \rtimes \mathrm{GL}(n, \mathbb{R})$. Recently this was generalized to almost crystallographic groups, see [1], [3] and [4].

We shall generalize this result to all homomorphisms (not necessarily isomorphisms). Topologically, this implies that every continuous map on an infra-nilmanifold is homotopic to a map induced by an affine endomorphism on the Lie group level. It can be stated as: every endomorphism of an almost crystallographic group is semiconjugate to an affine endomorphism.

THEOREM 1.1. Let $\pi, \pi^{\prime} \subset \operatorname{Aff}(G)$ be two almost crystallographic groups. Then for any homomorphism $\theta: \pi \rightarrow \pi^{\prime}$, there exists $g=$ $(d, D) \in \operatorname{aff}(G)$ such that $\theta(\alpha) \cdot g=g \cdot \alpha$ for all $\alpha \in \pi$.

Corollary 1.2. Let $M=\pi \backslash G$ be an infra-nilmanifold, and $h: M \rightarrow M$ be any map. Then $h$ is homotopic to a map induced from an affine endomorphism $G \rightarrow G$.

COROllaRY $1.3[\mathbf{3}, 4]$. Homotopy equivalent infra-nilmanifolds are affinely diffeomorphic.

Now we consider the uniqueness problem: How many $g$ 's are there? Let $\Phi=\pi /(G \cap \pi) \subset \operatorname{Aut}(G)$ and $\Phi^{\prime}=\pi^{\prime} /\left(G \cap \pi^{\prime}\right) \subset \operatorname{Aut}(G)$ be the holonomy groups of $\pi$ and $\pi^{\prime}$. Let $\Psi^{\prime}$ be the image of $\theta(\pi)$ in $\Phi^{\prime}$. So $\Phi^{\prime} \subset \operatorname{Aut}(G)$. Let $G^{\Psi^{\prime}}$ denote the fixed point set of the action. For $c \in G, \mu(c)$ denotes conjugation by $c$. Therefore, $\mu(c)(x)=c x c^{-1}$ for all $x \in G$. The group of all inner automorphisms is denoted by $\operatorname{Inn}(G)$.

Proposition 1.4 (Uniqueness). With the same notation as above, suppose $\theta(\alpha) \cdot g=g \cdot \alpha$ for all $\alpha \in \pi$. Then $\theta(\alpha) \cdot \gamma=\gamma \cdot \alpha$ for all $\alpha \in \pi$ if and only if $\gamma=\xi \cdot g$, where $\xi=\left(c, \mu\left(c^{-1}\right)\right)$, for $c \in G^{\Psi^{\prime}}$. Therefore, $D$ is unique up to $\operatorname{Inn}(G)$. If $\theta$ is an isomorphism, then $c \in G^{\Phi^{\prime}}$. In particular, if $\pi$ is a Bieberbach group with $H^{1}(\pi ; \mathbb{R})=0$ and $\theta$ is an isomorphism, then such $a g$ is unique.

EXAMPLE 1.5. The subgroup $\Gamma=\pi \cap G$ of of an almost crystallographic group $\pi$ is characteristic, but not fully invariant. The 
homomorphism $\theta$ in the Theorem 1.1 may not map the maximal normal nilpotent subgroup $\Gamma$ of $\pi$ into that of $\pi^{\prime}$. This causes a lot of trouble. Let $\pi$ be an orientable 4-dimensional Bieberbach group with holonomy group $\mathbb{Z}_{2}$. More precisely, $\pi \subset \mathbb{R}^{4} \rtimes O(4)=$ $E(4) \subset \operatorname{Aff}\left(\mathbb{R}^{4}\right)$ is generated by $\left(e_{1}, I\right),\left(e_{2}, I\right),\left(e_{3}, I\right),\left(e_{4}, I\right)$ and $(a, A)$, where $a=(1 / 2,0,0,0)^{t}$, and $A$ is diagonal matrix with diagonal entries $1,-1,-1$ and 1 . Note that $(a, A)^{2}=\left(e_{1}, I\right)$. The subgroup generated by $\left(e_{1}, I\right),\left(e_{2}, I\right),\left(e_{3}, I\right)$, and $(a, A)$ forms a 3 dimensional Bieberbach group $\mathcal{G}_{2}$, and $\pi=\mathcal{G}_{2} \times \mathbb{Z}$. Consider the endomorphism $\theta: \pi \rightarrow \pi$ which is the composite $\pi \rightarrow \mathbb{Z} \rightarrow \pi$, where the first map is the projection onto $\mathbb{Z}=\left\langle\left(e_{4}, I\right)\right\rangle$ and the second map sends $\left(e_{4}, I\right)$ to $(a, A)$. Thus the homomorphism $\theta$ does not map the maximal normal abelian subgroup $\mathbb{Z}^{4}$ (generated by the 4 translations) into itself. Such a $\mathbb{Z}^{4}$ is characteristic but not fully invariant in $\pi$. Let

$$
d=\left[\begin{array}{l}
x \\
0 \\
0 \\
y
\end{array}\right], \quad D=\left[\begin{array}{cccc}
0 & 0 & 0 & 1 / 2 \\
0 & 0 & 0 & 0 \\
0 & 0 & 0 & 0 \\
0 & 0 & 0 & 0
\end{array}\right]
$$

and let $g=(d, D)$. Then it is easy to see $\theta(\alpha) \cdot g=g \cdot \alpha$ for all $\alpha \in \pi$.

According to the Proposition 1.4, the element $g=(d, D)$ is the most general form. The matrix $D$ is uniquely determined and the translation part $d$ can vary only in two dimensions.

Proof of Theorem 1.1. Let $\Gamma=\pi \cap G, \Gamma^{\prime}=\pi^{\prime} \cap G$. As the example shows, the characteristic subgroup $\Gamma$ may not go into $\Gamma^{\prime}$ by the homomorphism $\theta$. Let $\Lambda=\Gamma \cap \theta^{-1}\left(\Gamma^{\prime}\right)$. Then $\Lambda$ is a normal subgroup of $\pi$ and has a finite index. Let $Q=\pi / \Lambda$.

Consider the homomorphism $\Lambda \rightarrow \Gamma^{\prime} \hookrightarrow G$, where the first map is the restriction of $\theta$. Since $\Lambda$ is a lattice of $G$, by Malćev's work, any such a homomorphism extends uniquely to a continuous homomorphism $C: G \rightarrow G$, cf. $[5,2.11]$. Thus, $\left.\theta\right|_{\Lambda}=\left.C\right|_{\Lambda}$, where $C \in \operatorname{Endo}(G)$; and hence, $\theta(z, 1)=(C z, 1)$ for all $z \in \Lambda$ (more precisely, $(z, 1) \in \Lambda)$.

Let us denote the composite homomorphism $\pi \rightarrow \pi^{\prime} \rightarrow G \rtimes$ $\operatorname{Aut}(G) \rightarrow \operatorname{Aut}(G)$ by $\bar{\theta}$; and define a map $f: \pi \rightarrow G$ by

$$
\theta(w, K)=(C w \cdot f(w, K), \bar{\theta}(w, K)) .
$$


For any $(z, 1) \in \Lambda$ and $(w, K) \in \pi$, apply $\theta$ to both sides of $(w, K)(z, 1)(w, K)^{-1}=\left(w \cdot K z \cdot w^{-1}, 1\right)$ to get $C w \cdot f(w, K)$. $\bar{\theta}(w, K)(C z) \cdot f(w, K)^{-1} \cdot(C w)^{-1}=\theta\left(w \cdot K z \cdot w^{-1}\right)$. However, $w \cdot K z \cdot w^{-1} \in \Lambda$ since $\Lambda$ is normal in $\pi$, and the latter term equals to $C\left(w \cdot K z \cdot w^{-1}\right)=C w \cdot C K z \cdot(C w)^{-1}$ since $C: G \rightarrow G$ is a homomorphism. From this we have

$$
\bar{\theta}(w, K)(C z)=f(w, K)^{-1} \cdot C K z \cdot f(w, K) .
$$

This is true for all $z \in \Lambda$. Note that $\bar{\theta}(w, K)$ and $K$ are automorphisms of the Lie group $G$; and $C: G \rightarrow G$ is an endomorphism. By the uniqueness of extension of a homomorphism $\Lambda \rightarrow G$ to an endomorphism $G \rightarrow G$, as mentioned above, the equality (2) holds true for all $z \in G$. It is also easy to see that $f(z w, K)=f(w, K)$ for all $z \in \Lambda$ so that $f: \pi \rightarrow G$ does not depend on $\Lambda$. Thus, $f$ factors through $Q=\pi / \Lambda$. Moreover, $\bar{\theta}: \pi \rightarrow \operatorname{Aut}(G)$ also factors through $Q$ since $\Lambda$ maps trivially into $\operatorname{Aut}(G)$. We still use the notation $(w, K)$ to denote elements of $Q$ and $\bar{\theta}$ to denote the induced map $Q \rightarrow \operatorname{Aut}(G)$.

Claim. With the $Q$-structure on $G$ via $\bar{\theta}: Q \rightarrow \operatorname{Aut}(G), f \in$ $Z^{1}(Q ; G)$; that is $f: Q \rightarrow G$ is a crossed homomorphism.

Proof. We shall show

$$
f\left((w, K) \cdot\left(w^{\prime}, K^{\prime}\right)\right)=f(w, K) \cdot \bar{\theta}(w, K) f\left(w^{\prime}, K^{\prime}\right)
$$

for all $(w, K),\left(w^{\prime}, K^{\prime}\right) \in \pi$. (Note that we are using the elements of $\pi$ to denote the elements of $Q$.) Apply $\theta$ to both sides of $(w, K)\left(w^{\prime}, K^{\prime}\right)=\left(w \cdot K w^{\prime}, K K^{\prime}\right)$ to get $C w \cdot f(w, K) \cdot \bar{\theta}(w, K)\left[C w^{\prime}\right.$. $\left.f\left(w^{\prime}, K^{\prime}\right)\right]=C\left(w \cdot K w^{\prime}\right) \cdot f\left((w, K)\left(w^{\prime}, K^{\prime}\right)\right)$. From this it follows that

$$
\begin{aligned}
f\left((w, K)\left(w^{\prime}, K^{\prime}\right)\right)=\left(C K w^{\prime}\right)^{-1} \cdot f(w, K) \\
\cdot \bar{\theta}(w, K)\left(C w^{\prime}\right) \cdot \bar{\theta}(w, K) f\left(w^{\prime}, K^{\prime}\right) .
\end{aligned}
$$

From (2) we have $\bar{\theta}(w, K) C w^{\prime}=f(w, K)^{-1} \cdot C K w^{\prime} \cdot f(w, K)$ so that $f\left((w, K) \cdot\left(w^{\prime}, K^{\prime}\right)\right)=f(w, K) \cdot \bar{\theta}(w, K) f\left(w^{\prime}, K^{\prime}\right)$.

In [4], it was proved that $H^{1}(Q ; G)=0$ whenever $Q$ is a finite group and $G$ is a connected and simply connected nilpotent Lie 
group. The proof uses induction on the nilpotency of $G$ together with the fact that $H^{1}(Q ; G)=0$ for a finite group $Q$ and a real vector group $G$. This means that any crossed homomorphism is "principal". In other words, there exists $d \in G$ such that

$$
f(w, K)=d \cdot \bar{\theta}(w, K)\left(d^{-1}\right) .
$$

Let $D=\mu\left(d^{-1}\right) \circ C$ and $g=(d, D) \in \operatorname{aff}(G)$, and we check that $\theta$ is "conjugation" by $g$. Using (1), (2) and (3), one can show $\bar{\theta}(w, K) \circ \mu\left(d^{-1}\right) \circ C=\mu\left(d^{-1}\right) \circ C \circ K$. Thus, for any $(w, K) \in \pi$,

$$
\begin{aligned}
\theta & (w, K) \cdot(d, D) \\
& =(C w \cdot f(w, K), \bar{\theta}(w, K)) \cdot\left(d, \mu\left(d^{-1}\right) \circ C\right) \\
& =\left(C w \cdot f(w, K) \cdot \bar{\theta}(w, K)(d), \bar{\theta}(w, K) \circ \mu\left(d^{-1}\right) \circ C\right) \\
& =\left(C w \cdot d \cdot \bar{\theta}(w, K)\left(d^{-1}\right) \cdot \bar{\theta}(w, K)(d), \bar{\theta}(w, K) \circ \mu\left(d^{-1}\right) \circ C\right) \\
& =\left(C w \cdot d, \mu\left(d^{-1}\right) \circ C \circ K\right) \\
& =(d, D) \cdot(w, K) .
\end{aligned}
$$

This finishes the proof of theorem.

Proof of Corollary 1.2. We start with the homomorphism $h_{\#}$ : $\pi_{1}(M) \rightarrow \pi_{1}(M)$, induced from $h$, as our $\theta$ in the Theorem 1.1 and obtain $\tilde{g}=(d, D)$ satisfying

$$
h_{\#}(\alpha) \circ \tilde{g}=\tilde{g} \circ \alpha .
$$

Let $g: M \rightarrow M$ be the induced map. Then $h_{\#}=g_{\#}$. Since any two continuous maps on a closed aspherical manifold inducing the same homomorphism on the fundamental group (up to conjugation by an element of the fundamental group) are homotopic to each other, $h$ is homotopic to $g$. This completes the proof of the corollary.

Proof of Proposition 1.4. Let $g=(d, D), \gamma=(c, C)$. Since $\theta(\alpha) \cdot g=g \cdot \alpha$ holds when $\alpha=(z, 1) \in \Lambda$, we have $D z=$ $d^{-1} z^{\prime} d$, where $\theta(z, 1)=\left(z^{\prime}, 1\right)$. Similarly, $C z=c^{-1} z^{\prime} c$. Thus $C z=\mu\left(c^{-1} d\right) D z$ for all $z \in \Lambda$. Since $\Lambda$ is a lattice, this equality holds on $G$. Consequently, $C=\mu\left(c^{-1} d\right) D$. Now $\gamma=(c, C)=$ $\left(c, \mu\left(c^{-1} d\right) D\right)=\left(d^{-1} c, \mu\left(c^{-1} d\right)\right)(d, D)=\left(h, \mu\left(h^{-1}\right)\right)(d, D)$, if we 
let $h=d^{-1} c$. Set $\xi=\left(h, \mu\left(h^{-1}\right)\right)$. Then $\gamma=\xi \cdot g$. Now we shall observe that $h \in G^{\Psi^{\prime}}$. Let $\theta(\alpha)=(b, B)$. Then $\theta(\alpha) \xi g=\theta(\alpha) \gamma=$ $\gamma \alpha=\xi g \alpha=\xi \theta(\alpha) g$ yields $B h=h$ for all $(b, B)=\theta(\alpha)$. Clearly then $B \in \Psi^{\prime}$ by definition. For a Bieberbach group $\pi$, note that $\operatorname{rank} H^{1}(\pi ; \mathbb{Z})=\operatorname{dim} G^{\Phi}$.

2. Application to Fixed-point theory. Let $M$ be a closed manifold and let $f: M \rightarrow M$ be a continuous map. The Lefschetz number $L(f)$ of $f$ is defined by

$$
L(f):=\sum_{k} \operatorname{trace}\left\{\left(f_{*}\right)_{k}: H_{k}(M ; \mathbb{Q}) \rightarrow H_{k}(M ; \mathbb{Q})\right\}
$$

To define the Nielsen number $N(f)$ of $f$, we define an equivalence relation on $\operatorname{Fix}(f)$ as follows: For $x_{0}, x_{1} \in \operatorname{Fix}(f), x_{0} \sim x_{1}$ if and only if there exists a path $c$ from $x_{0}$ to $x_{1}$ such that $c$ is homotopic to $f \circ c$ relative to the end points. An equivalence class of this relation is called a fixed point class $(=\mathrm{FPC})$ of $f$. To each FPC $F$, one can assign an integer ind $(f, F)$. A FPC $F$ is called essential if $\operatorname{ind}(f, F) \neq 0$. Now,

$N(f):=$ the number of essential fixed point classes.

These two numbers give information on the existence of fixed point sets. If $L(f) \neq 0$, every self-map $g$ of $M$ homotopic to $f$ has a non-empty fixed point set. The Nielsen number is a lower bound for the number of components of the fixed point set of all maps homotopic to $f$. Even though $N(f)$ gives more information than $L(f)$ does, it is harder to calculate. If $M$ is an infra-nilmanifold, and $f$ is homotopically periodic, then it is known that $L(f)=N(f)$.

Lemma 2.1. Let $B \in \mathrm{GL}(n, \mathbb{R})$ with a finite order. Then $\operatorname{det}(I-$ B) $\geq 0$.

Proof. Since $B$ has finite order, it can be conjugated into the orthogonal group $O(n)$. Since all eigenvalues are roots of unity, there exists $P \in \mathrm{GL}(n, \mathbb{R})$ such that $P B P^{-1}$ is a block diagonal matrix, with each block being a $1 \times 1$, or, a $2 \times 2$-matrix. All $1 \times 1$ blocks must be $D=[ \pm 1]$, and hence $\operatorname{det}(I-D)=0$ or 2 . For a 
$2 \times 2$ block, it is of the form $\left[\begin{array}{cc}\cos t & \sin t \\ -\sin t \cos t\end{array}\right]$. Consequently, each $2 \times 2$ block $D$ has the property that $\operatorname{det}(I-D)=(1-\cos t)^{2}+\sin ^{2} t=$ $2(1-\cos t) \geq 0$.

THEOREM 2.2. Let $f: M \rightarrow M$ be a continuous map on an infra-nilmanifold $M=\pi \backslash G$. Let $g=(d, D) \in \operatorname{aff}(G)$ be a homotopy lift of $f$ by Corollary 1.2. Then $L(f)=N(f)$ (resp., $L(f)=$ $-N(f))$ if and only if $\operatorname{det}\left(I-D_{*} A_{*}\right) \geq 0\left(\right.$ resp., $\left.\operatorname{det}\left(I-D_{*} A_{*}\right) \leq 0\right)$ for all $A \in \Phi$, the holonomy group of $M$.

Proof. Since $L(f)$ and $N(f)$ are homotopy invariants, we may assume that $f=g$. Let $\Gamma=\pi \cap G$, and let $\Lambda=\Gamma \cap f_{\#}^{-1} f_{\#}\left(\Gamma \cap f_{\#}^{-1}(\Gamma)\right)$. Then $\Gamma$ is a normal subgroup of $\pi$, of finite index. Moreover, $f_{\#}: \pi \rightarrow \pi$ maps $\Lambda$ into itself. Therefore, $f$ induces a map on the finite-sheeted regular covering space $\Lambda \backslash G$ of $\pi \backslash G$.

Let $\tilde{f}$ be a lift of $f$ to $\Gamma \backslash G$. Then

$$
\begin{aligned}
L(f) & =\frac{1}{[\pi: \Lambda]} \Sigma \operatorname{ind}\left(f, p_{\Lambda} \operatorname{Fix}(\alpha \widetilde{f})\right) \\
N(f) & =\frac{1}{[\pi: \Lambda]} \Sigma\left|\operatorname{ind}\left(f, p_{\Lambda} \operatorname{Fix}(\alpha \widetilde{f})\right)\right|
\end{aligned}
$$

where the sum ranges over all $\alpha \in \pi / \Lambda$. See, [2, III 2.12]. However, each $\alpha \tilde{f}$ is a map on the nilmanifold $\Lambda \backslash G$, and hence $\operatorname{ind}\left(f, p_{\Lambda} \operatorname{Fix}(\alpha \widetilde{f})\right)$ is determined by $\operatorname{det}\left(I-(\alpha f)_{*}\right)$. It is not hard to see that, for any $\alpha \in \operatorname{Inn}(G), \alpha_{*}$ has eigenvalue only 1 . Therefore, it is enough to look at elements with non-trivial holonomy. Now the hypothesis guarantees that $\operatorname{det}\left(I-(\alpha f)_{*}\right)=\operatorname{det}\left(I-D_{*} A_{*}\right) \geq 0$ or $\leq 0$ always. Consequently, $L(f)=N(f)$ or $L(f)=-N(f)$.

Conversely, suppose $L(f)=N(f)$ (resp. $L(f)=-N(f)$ ). Let $\alpha=(a, A) \in \pi$. If $\operatorname{Fix}(g \circ \alpha)=\varnothing$, then clearly $\operatorname{det}\left(I-D_{*} A_{*}\right)=0$. Otherwise, $\operatorname{Fix}(g \circ \alpha)$ is isolated, and the indices at these fixed points are $\operatorname{det}\left(I-D_{*} A_{*}\right)$. By the formula above relating $L(f), N(f)$ with the ones on covering spaces, all $\operatorname{det}\left(I-D_{*} A_{*}\right)$ must have the same sign. This proves the theorem.

CoROllaRY 2.3 [3]. Let $f: M \rightarrow M$ be a homotopically periodic map on an infra-nilmanifold. Then $N(f)=L(f)$.

Proof. Here is an argument which is completely different from the one in [3]. Let $\Gamma=\pi \cap G$, and $\Phi=\pi / \Gamma$, the holonomy group. 
Let $g=(d, D) \in G \rtimes \operatorname{Aut}(G)$ be a homotopy lift of $f$ to $G$. Let $E$ be the lifting group of the action of $\langle g\rangle$ to $G$. That is, $E$ is generated by $\pi$ and $g$. Then $E / \Gamma$ is a finite group generated by $\Phi$ and $D$. For every $A \in \Phi, D A$ lies in $E / \Gamma$, and has a finite order. By Lemma $2.1, \operatorname{det}(I-D A) \geq 0$ for all $A \in \Phi$. By Theorem 2.2, $L(f)=N(f)$.

Let $S$ be a connected, simply connected solvable Lie group and $H$ be a closed subgroup of $S$. The coset space $H \backslash S$ is called a solvmanifold.

Corollary 2.4 [7]. Let $f: M \rightarrow M$ be a homotopically periodic map on an infra-solvmanifold. Then $N(f)=L(f)$.

Proof. In [5], the statement for solvmanifolds was proved. We needed a subgroup invariant under $f_{\#}$. To achieve this, a new model space $M^{\prime}$ which is homotopy equivalent to $M$, together with a map $f^{\prime}: M^{\prime} \rightarrow M^{\prime}$ corresponding to $f$ was constructed. The new space $M^{\prime}$ is a fiber bundle over a torus with fiber a nilmanifold; and $f^{\prime}$ is fiber-preserving. Moreover, we found a fully invariant subgroup $\Lambda$ of $\pi$ of finite index (so, is invariant under $f_{\#}^{\prime}$ ). Now we can apply the same argument as in the proof of Theorem 2.2.

EXAMPLE 2.5. Let $\pi$ be an orientable 3-dimensional Bieberbach group with holonomy group $\mathbb{Z}_{2}$. More precisely, $\pi \subset \mathbb{R}^{3} \rtimes O(3)=$ $E(3)$ is generated by $\left(e_{1}, I\right),\left(e_{2}, I\right),\left(e_{3}, I\right)$ and $(a, A)$, where $a=$ $(1 / 2,0,0)^{t}, A$ is a diagonal matrix with diagonal entries $1,-1$ and -1 . Note that $(a, A)^{2}=\left(e_{1}, I\right)$. Let $M=\mathbb{R}^{3} / \pi$ be the flat manifold. Consider the endomorphism $\theta: \pi \rightarrow \pi$ which is defined by the conjugation by $g=(d, D)$, where

$$
d=\left[\begin{array}{l}
0 \\
0 \\
0
\end{array}\right], \quad D=\left[\begin{array}{lll}
3 & 0 & 0 \\
0 & 0 & 1 \\
0 & 2 & 0
\end{array}\right] \text {. }
$$

Let $f: M \rightarrow M$ be the map induced from $g$. There are only two conjugacy classes of $g$; namely, $g$ and $\alpha g$. $\operatorname{Fix}(g)=(0,0,0)^{t}$ and $\operatorname{Fix}(\alpha g)=(1 / 4,0,0)^{t}$. Since $\operatorname{det}(I-D)=\operatorname{det}(I-A D)=+2$, $L(f)=N(f)=2$.

The Lefschetz number can be calculated from homology groups also.

(1) $H_{0}(M ; \mathbb{R})=\mathbb{R} ; f_{*}$ is the identity map. 
(2) $\quad H_{1}(M ; \mathbb{R})=\mathbb{R}$, which is generated by the element $\left(e_{1}, I\right)$. $f_{*}$ is multiplication by 3 (the $(1,1)$-entry of $D$ ).

(3) $H_{2}(M ; \mathbb{R})=\mathbb{R} ; f_{*}$ is multiplication by $\operatorname{det}\left[\begin{array}{ll}0 & 1 \\ 2 & 0\end{array}\right]=-2$.

(4) $H_{3}(M ; \mathbb{R})=\mathbb{R} ; f_{*}$ is multiplication by $\operatorname{det}(D)=-6$. Therefore, $L(f)=\Sigma(-1)^{i} \operatorname{trace} f_{*}=1-3+(-2)-(-6)=2$. Note that $f$ has infinite period, and this example is not covered by Corollary 2.3.

EXAMPLE 2.6. Let $\pi$ be same as in Example 2.5. This time $g=(d, D)$, is given by

$$
d=\left[\begin{array}{l}
0 \\
0 \\
0
\end{array}\right], \quad D=\left[\begin{array}{lll}
3 & 0 & 0 \\
0 & 1 & 1 \\
0 & 1 & 2
\end{array}\right]
$$

Let $f: M \rightarrow M$ be the map induced from $g$. There are six conjugacy classes of $g$; namely, $g$ and $\alpha g, \alpha t_{1} g, \alpha t_{1}^{2} g, \alpha t_{1}^{3} g$, and $\alpha t_{1}^{4} g$. Each class has exactly one fixed point. Clearly, $\operatorname{det}(I-D)=+2$ and $\operatorname{det}(I-A D)=-10$. Therefore, the first fixed point has index +1 and the rest have index -1 . Consequently, $L(f)=-4$, while $N(f)=6$.

\section{REFERENCES}

[1] L. Auslander, Bieberbach's theorems on space groups and discrete uniform subgroups of Lie groups, Annals of Math., 71 (1960), 579-590.

[2] B. J. Jiang, Lectures on Nielsen fixed point theory, Contemporary Mathematics 14, A.M.S., vol 14, 1983.

[3] S. Kwasik and K. B. Lee, Nielsen numbers of homotopically periodic maps on infra-nilmanifolds, J. London Math. Soc., (2) 38 (1988), 544-554.

[4] Y. Kamishima, K. B. Lee and F. Raymond, The Seifert construction and its applications to infranilmanifolds, Quarterly J. Math. (Oxford), 34 (1983), 433-452.

[5] K. B. Lee, Nielsen Numbers of periodic maps on Solvmanifolds, Proc. Amer. Math. Soc., 116 (1992), 575-579.

[6] K. B. Lee and F. Raymond, Rigidity of almost crystallographic groups, Contemp. Math. Amer. Math. Soc., 44 (1985), 73-78.

[7] C. McCord, Nielsen numbers of homotopically periodic maps on infrasolvmanifolds, Proc. Amer. Math. Soc., 120 (1994), 311-315.

[8] M. S. Raghunathan, Discrete Subgroups of Lie Groups, Springer, 1972. 
Received July 14, 1992.

UNIVERSITY OF OKLAHOMA

NORMAN, OK 73019

E-mail address: kblee@dstn3.math.uoknor.edu 


\section{PACIFIC JOURNAL OF MATHEMATICS}

Founded by E. F. Beckenbach (1906-1982) and F. Wolf (1904-1989)

\section{EDITORS}

Sun-Yung Alice Chang (Managing Editor)

University of California

Los Angeles, CA 90095-1555

pacific@math.ucla.edu

F. Michael Christ

University of California

Los Angeles, CA 90095-1555

christ@math.ucla.edu

Thomas Enright

University of California

San Diego, La Jolla, CA 92093

tenright@ucsd.edu

Nicholas Ercolani

University of Arizona

Tucson, AZ 85721

ercolani@math.arizona.edu
Robert Finn

Stanford University

Stanford, CA 94305

finn@gauss.stanford.edu

Vaughan F. R. Jones

University of California

Berkeley, CA 94720

vfr@math.berkeley.edu

Steven Kerckhoff

Stanford University

Stanford, CA 94305

spk@gauss.stanford.edu

\author{
Martin Scharlemann \\ University of California \\ Santa Barbara, CA 93106 \\ mgscharl@math.ucsb.edu \\ Gang Tian \\ Courant Institute \\ New York University \\ New York, NY 10012-1100 \\ tiang@taotao.cims.nyu.edu
V. S. Varadarajan
University of California
Los Angeles, CA 90095-1555
vsv@math.ucla.edu

\section{SUPPORTING INSTITUTIONS}

\section{CALIFORNIA INSTITUTE OF TECHNOLOGY NEW MEXICO STATE UNIVERSITY OREGON STATE UNIVERSITY \\ STANFORD UNIVERSITY \\ UNIVERSITY OF ARIZONA \\ UNIVERSITY OF BRITISH COLUMBIA \\ UNIVERSITY OF CALIFORNIA UNIVERSITY OF HAWAII}

\author{
UNIVERSITY OF MONTANA \\ UNIVERSITY OF NEVADA, RENO \\ UNIVERSITY OF OREGON \\ UNIVERSITY OF SOUTHERN CALIFORNIA \\ UNIVERSITY OF UTAH \\ UNIVERSITY OF WASHINGTON \\ WASHINGTON STATE UNIVERSITY
}

The supporting Institutions listed above contribute to the cost of publication of this Journal, but they are not owners or publishers and have no responsibility for its contents or policies.

Manuscripts must be prepared in accordance with the instructions provided on the inside back cover.

The Pacific Journal of Mathematics (ISSN 0030-8730) is published monthly except for July and August. Regular subscription rate: $\$ 215.00$ a year (10 issues). Special rate: $\$ 108.00$ a year to individual members of supporting institutions.

Subscriptions, orders for back issues published within the last three years, and changes of subscribers address should be sent to Pacific Journal of Mathematics, P.O. Box 4163, Berkeley, CA 94704-0163, U.S.A. Prior back issues are obtainable from Kraus Periodicals Co., Route 100, Millwood, NY 10546.

The Pacific Journal of Mathematics at the University of California, c/o Department of Mathematics, 981 Evans Hall, Berkeley, CA 94720 (ISSN 0030-8730) is published monthly except for July and August. Second-class postage paid at Berkeley, CA 94704, and additional mailing offices. POSTMASTER: send address changes to Pacific Journal of Mathematics, P.O. Box 6143, Berkeley, CA 94704-0163.

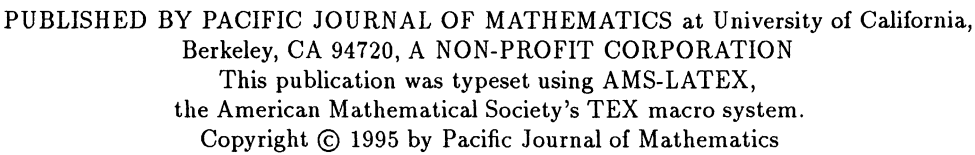




\section{PACIFIC JOURNAL OF MATHEMATICS}

Volume $168 \quad$ No. $1 \quad$ March 1995

Compact contractible $n$-manifolds have arc spines $(n \geq 5)$

FREDRIC DAVIS ANCEL and CRAIG R. GUILBAULT

On uniform homeomorphisms of the unit spheres of certain Banach lattices

\section{FOUAD CHAATIT}

Applications of subordination chains to starlike mappings in $\mathbb{C}^{n}$

MARTIN CHUAQUi

The $L^{p}$ theory of standard homomorphisms

FEREIDOUn GHAHRAMANI and SANDY GRABINER

Metrics for singular analytic spaces

Caroline Galt Grant and Pierre Milman

Maps on infra-nilmanifolds

KYUNG BAI LEE

Hardy spaces and oscillatory singular integrals. II

YIBIAO PAN

Continuity of convex hull boundaries

Linda Jo Goldway KeEn and Caroline SERIES 